\title{
Confocal Laser Endomicroscopy: Applications in Clinical and Translational Science-A Comprehensive Review
}

\author{
Martin Goetz \\ Innere Medizin I, Universitätsklinikum Tübingen, 72076 Tübingen, Germany \\ Correspondence should be addressed to Martin Goetz, martin.goetz@med.uni-tuebingen.de \\ Received 7 November 2012; Accepted 11 December 2012 \\ Academic Editors: V. J. Amatya, R. Drut, T. Kovács, and A. Wincewicz \\ Copyright () 2012 Martin Goetz. This is an open access article distributed under the Creative Commons Attribution License, which \\ permits unrestricted use, distribution, and reproduction in any medium, provided the original work is properly cited. \\ Confocal laser endomicroscopy (CLE) is a novel tool in the endoscopist's armamentarium. It allows on-site histological \\ information. The ability of gastroenterologists to interpret such microscopic information has been demonstrated in multiple \\ studies from the upper and lower gastrointestinal tract. Recently, the field of application has expanded to provide hepatobiliary and \\ intra-abdominal CLE imaging. CLE allows "smart," targeted biopsies and is able to guide endoscopic interventions. But CLE is also \\ translational in its approach and permits functional imaging that significantly impacts on our understanding of gastrointestinal \\ diseases. Molecular imaging with CLE allows detection and characterization of lesions and may even be used for prediction of \\ response to targeted therapy. This paper provides a comprehensive review over current applications of CLE in clinical applications \\ and translational science.
}

\section{Introduction}

Confocal laser endomicroscopy (CLE) is a novel endoscopic method that permits on-site microscopy of the gastrointestinal mucosa after the application of a fluorescent agent. Since the first description in 2004 [1], the number of diseases studied with this technique has been steadily growing [2-9]. Trials with CLE have moved from feasibility studies in different parts of the endoscopically accessible areas of the gastrointestinal tract towards inflammatory and (pre-) neoplastic diseases that are often only incompletely appreciated by random biopsies. Here, CLE is the tool to enable "smart" biopsies, biopsies that are targeted to areas of interest by means of intravital microscopy. But indications have broadened from the upper and lower GI tract towards intravital microscopy of the biliary tract, the liver or pancreas. In addition, endoscopic disciplines outside the field of gastroenterology have evaluated CLE within their field. The option of intravital microscopy makes CLE an ideal tool to study pathophysiological events dynamically in their natural surroundings in patients. Labeling of molecular targets links clinical patient care to translational science.

\section{Technique of Endomicroscopy and Staining Protocols}

2.1. Technique of Confocal Endomicroscopy. Light microscopy uses white light in the visible range and lens systems for magnification. This necessitates incident light shining through thin, translucent tissue section in bench top light microscopy. For endoscopy, ultrahigh magnification endoscopes have been developed ("Endocytoscopy") that deliver high resolution images of the uppermost epithelial layer of the gastrointestinal tract. With different staining techniques, in vivo microscopy of superficial cells becomes possible [1012].

Confocal laser endomicroscopy (CLE) is substantially different. A low-power laser is used to focus onto a single point within the tissue. Light emanating from this point is focused through a pinhole to a detector while the laser raster scans the two-dimensional imaging plane, whereas light from outside this focally illuminated point is geometrically rejected and does therefore not blur the resultant image. This allows imaging at the tissue surface, but also below 
the surface without the need to physically disrupt the tissue integrity.

Two systems are currently marketed for CLE: the first system to receive EMA- and FDA-approval was an endoscopeintegrated (eCLE) system where a miniaturized scanner (Optiscan, Notting Hill, Australia) has been integrated into the distal tip of a conventional colonoscope (distal tip diameter 13.2 mm; Pentax EC-3870CIFK, Tokyo, Japan). A single optical fibre is mounted into a magnetic tuning fork, raster scanning the tissue. It serves as a pinhole for CLE and delivers a low-energy excitation wavelength of $488 \mathrm{~nm}$. The collection bandwidth is 505 to $585 \mathrm{~nm}$. The imaging plane depth can be user-adjusted from surface to $250 \mu \mathrm{m}$. Serial optical sections are obtained parallel to the tissue surface within a FOV of $475 \times 475 \mu \mathrm{m}$ and with a resolution of $1024 \times 1024$ pixels (lateral resolution of $0.7 \mu \mathrm{m}$ ). The same scanner was integrated into a handheld rigid probe (FIVE1, Optiscan) for laboratory or laparoscopic research. Prototypes have used near infrared excitation at $780 \mathrm{~nm}$ in patients for deeper tissue penetration.

The second system uses a flexible fibre bundle that can be passed through the working channel of most endoscopes (probe-based CLE, pCLE, Cellvizio, Mauna Kea Technology, Paris, France). In pCLE, the imaging plane depth is fixed, and resolution is somewhat lower (limited by the number of fibres). Image acquisition is faster, and real-time microscopic video sequences can be generated. Probes are available with different fields of view and diameter that allows probes to even be passed into the bile duct or through a needle positioned by endoscopic ultrasound. For animal research, a longer excitation wavelength $(660 \mathrm{~nm})$ is available in addition to excitation at $488 \mathrm{~nm}$ for the probes used in clinical practice.

2.2. In Vivo Imaging and Staining Protocols. As in bench top laser scanning microscopic, CLE relies on induced fluorescence. In patients, most studies used fluorescein as a fluorescent agent which has a favorable safety profile [13]. After intravenous injection, fluorescein rapidly diffuses throughout the body and provides high resolution images within seconds. Tissue architecture and vessels can be nicely seen, however nuclei are not stained. As an alternative, topical fluorescent agents have been studied. Acriflavine results in positive nuclear staining $[1,14]$. Therefore a theoretical risk of mutagenesis has been discussed, although acriflavine and similar compounds are constituents of many skin disinfectants and no such adverse events have been described so far. Nevertheless, conservative use is mandated by most CLE users. Cresyl violet has been studied as an alternative for simultaneous chromoendoscopy and immediate characterization by eCLE. Although the excitation and emission wavelengths do not peek within the above given range, the broad spectrum of cresyl violet still resulted in indirect nuclear visualization by cytoplasmic enrichment of the dye [15]. In animal studies with lower regulatory requirements a multitude of staining protocols have been evaluated in vivo [16], and even antibodies have been fluorescently labeled.
Similar to every advanced endoscopy technique, CLE requires thorough training. Such training has to cover two compounds. The technical aspect of targeting the probe (pCLE) or the slightly protruding confocal imaging window of eCLE onto the region of interest must be practiced. Some users of pCLE use a transparent cap to stabilize the probe on a lesion. The other training aspect is acquisition of a thorough knowledge of mucosal histopathology which is not included in the curriculum of most gastroenterologists. Close collaboration and feedback from an expert GI pathologist is very helpful in difficult cases. In contrast to conventional histopathology, CLE provides en-face sections through the mucosa, parallel to the tissue surface. Easy-touse classification systems have been established for a basic differentiation of normal from hyperplastic or inflammatory from neoplastic mucosal changes, demonstrating the trained gastroenterologists are able to use microscopy for immediate diagnosis at tissue level during ongoing endoscopy. From a study on Barrett's esophagus it has been estimated that after initial training approximately 100 cases are necessary for reliable eCLE diagnosis in patients [17]. However, for a first basic step to handle the CLE endoscope and to differentiate normal from abnormal tissue, a number of 30 examinations have been deemed sufficient, with online and printed support $[18,19]$. With video support and a learning set of typical images, a rapid learning curve has been demonstrated for pCLE even for nonexpert gastroenterologists [20].

CLE only covers a small mucosal area, pan-endomicroscopy of the GI surface is not feasible. Therefore the regions to be investigated by this point-technique have to be delineated by white light endoscopy (e.g., with high definition endoscopes) or by chromoendoscopy, which helps to unmask suspicious areas within the GI tract. The advantage of CLE during endoscopy is that normal tissue can be predicted with high accuracy and confidence. Therefore, random biopsies in diseases such as ulcerative colitis are no longer needed, if optical biopsies are judged by an expert endoscopist. Within larger lesions, the most suspicious part can be identified and targeted for physical tissue sampling. In studies, such a concept of microscopically targeted, "smart" biopsies has resulted in fewer biopsies, but higher yield to detect neoplasia in the upper [21] and lower GI tract [22].

In order to support on-site diagnosis for the gastroenterologist, automated image recognition tools have been evaluated. In pancreatic cancer xenografts imaged with pCLE, altered vessel tortuosity, density, and disorganization in malignant tissue have been found typical for the microvasculature of pancreatic ductal carcinoma. After application of a segmentation algorithm, a skeletonized image was submitted for fractal analysis [23]. Significantly increased microvessel density was also found in malignant tissue of Barrett's esophagus [24]. A content-based image retrieval, by using keywords or by filtering different fields for structured retrieval, was built on a case-based series to assist in diagnosis involving an algorithm for automatic feature extraction in eCLE images [25]. In a similar approach, a pCLE video retrieval atlas has been found helpful in delineating levels of expertise among CLE users [26]. 


\section{Upper Gastrointestinal Tract}

3.1. Endomicroscopy in the Esophagus. Following the above delineated method, eCLE was used in patients with suspected squamous cell cancer of the esophagus. First, multiple unstained areas were unmasked by chromoendoscopy with Lugol's solution. These 43 areas in 21 patients were then subjected to endomicroscopic characterization [27]. CLE correctly identified all neoplastic lesions based on typical aspects of cell and tissue architectural alterations. Two lesions were falsely classified as neoplastic. These results were confirmed in a follow-up trial, establishing irregular cellular arrangement, increased diameter, and irregular shape of intrapapillary capillary loops as diagnostic patterns [28].

Many studies on CLE have been conducted in Barrett's esophagus (Figure 1). This partly reflects the low confidence of gastroenterologists to detect preneoplastic lesions with the current state-of-the-art biopsy protocol ("Seattle protocol"), which is based on random quadrant biopsies, hoping to pick up neoplastic distributed with a patchy pattern within the area of intestinal metaplasia. On the other hand, once the suspicion of neoplasia has been raised, it is difficult to find the exact spot where the biopsies have been sampled. By providing on-site microscopy, CLE is able to directly guide resection during endoscopy. Typical changes consistent with Barrett's metaplasia are easily visible with CLE (Figure 1(a)). Goblet cells are identified by their dark mucin inclusion in the epithelium, and the villous structure and altered vasculature become visible after fluorescein injection. Neoplastic cells (Figure 1(b)) are commonly dark, potentially secondary to the fact that $\mathrm{pH}$ is lower in malignant cells (and fluorescein shows a pH-dependent fluorescence intensity). Vessels in Barrett's esophagus are clearly different from intrapapillary capillary loops in normal squamous epithelium. In a first trial, eCLE was able to accurately predict Barrett's metaplasia in over $90 \%$ of patients and to establish criteria for neoplastic changes [29]. A follow-up cross over study compared routine quadrant biopsies with biopsies targeted by eCLE. The per biopsy diagnostic yield was almost doubled by CLE, and two thirds of patients did not need any biopsy based on normal appearance of their Barrett's segment with CLE [21].

In further trials, pCLE showed a high negative predictive value (NPV) of $99 \%$, however at the price of a somewhat lower PPV of only 44\% [30]. Similar results were obtained from a trial in three expert centers assessing 68 patients with Barrett's esophagus. Compared to the Seattle biopsy protocol, pCLE had a high on-site NPV of 95\% to exclude neoplasia, but a low PPV of only 18\% [31]. A head-to-head comparison of eCLE versus pCLE to explain for differences in PPV and specificity has not been performed. This may be partly explained by the higher resolution of eCLE and visualisation of single cells to identify neoplasia, whereas the somewhat lower resolution of pCLE is sufficient for pattern recognition of nonneoplastic Barrett's glands. Future trials are thus necessary before random quadrant biopsies can be completely abandoned.

About half of the patients with reflux symptoms do not show endoscopic changes (nonerosive or endoscopically negative reflux disease, NERD). Objectification of these pa-

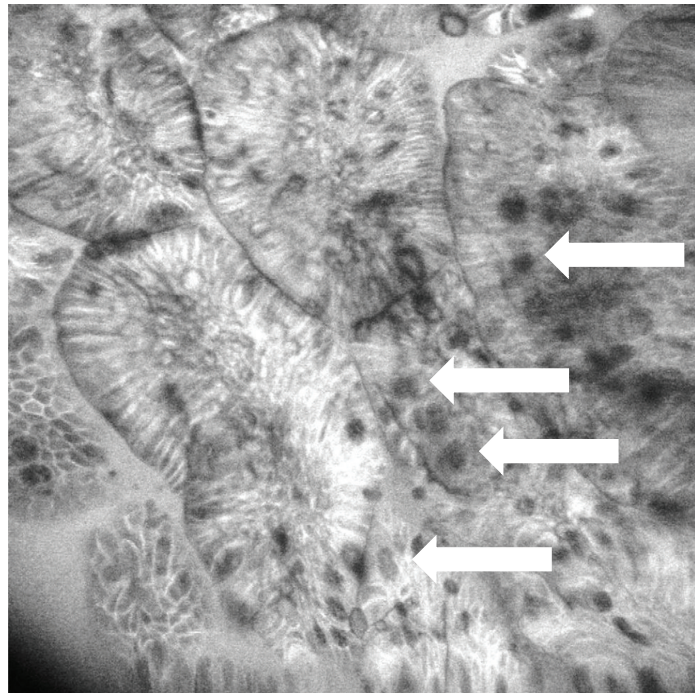

(a)

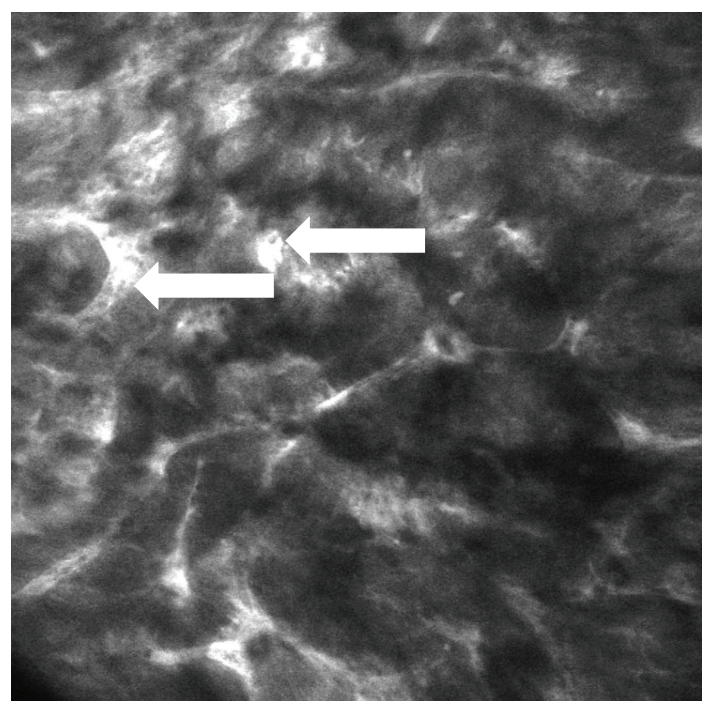

(b)

FIgURE 1: Barrett's esophagus. (a) In Barrett's esophagus, CLE is able to visualize goblet cells (arrows) by their black mucin inclusion. A double contour can be seen on the villous surface, corresponding to the brush border. (b) In another area (and deeper tissue section), crowding of irregular glands, irregular vessels (arrow), and leakage of fluorescein indicate the presence of neoplasia. Edge length is $475 \mu \mathrm{m}$.

tients' complaints is often difficult. In a recent trial, CLE was able to demonstrate that NERD patients had a significantly higher number and larger diameter of intrapapillary capillary loops per image than controls [32]. The intercellular spaces between squamous cells were found to be significantly dilated. This illustrates that "endoscopically negative" reflux may be endoscopically visible once appropriate imaging is applied (Figure 2).

3.2. Endomicroscopy of Gastric Pathologies. The healthy stomach shows a cobble stone pattern of the gastric epithelium (Figure 3). The gastric pits are round and crypt-like 


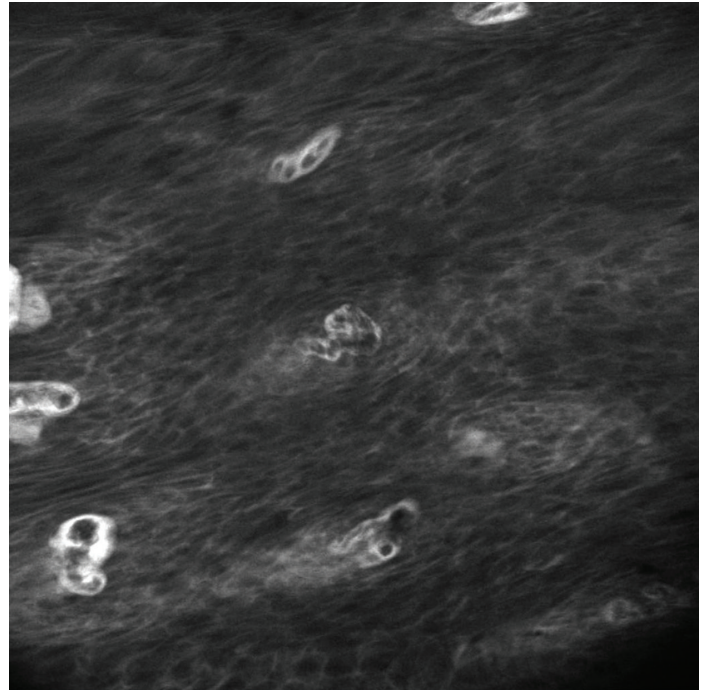

(a)

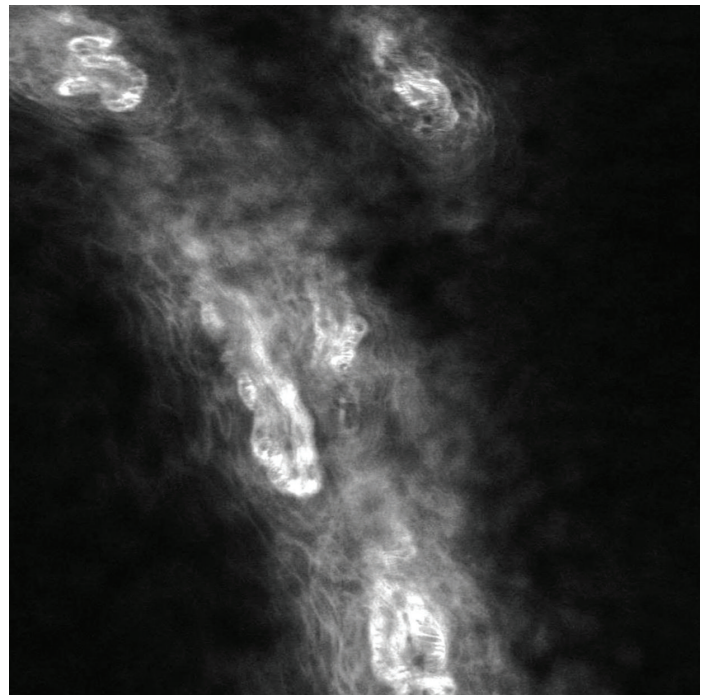

(b)

FIgure 2: Squamous cell mucosa of the esophagus. (a) In the normal esophagus, intrapapillary capillary loops can be seen at regular distance, and fluorescein leaks out to only a "halo" around each vessel loop. (b) In nonerosive reflux disease, IPCLs are elongated and dilated, single dark dots within the bright vessel lumina indicate red blood cells. Edge length is $475 \mu \mathrm{m}$.

in the corpus and fundus, and branched and slit-like in the antrum and the cardia [33]. Imaging in the stomach often requires high laser power due to the acidic milieu (see above). One of the earliest signs of gastritis is visualization of the high vascularity and leakage of fluorescein into the lumen, adding a functional aspect to microscopic imaging. However, since the deep aspects of the gastric glands are not visible with CLE and since inflammatory cells cannot be adequately differentiated with fluorescein due to the lack of nuclear visualization, classification of gastritis with CLE has not been a major focus of clinical research, but studies graded severity of gastritis [34].

Gastric neoplasia often arises in the setting of Helicobacter pylori-associated gastritis or gastric atrophy and intestinal metaplasia. These entities represent diffuse or patchy changes of the mucosa, and random biopsies often fail to cover all aspects of the mucosal alterations. Here, CLE is able to guide future management by taking multiple optical biopsies within few seconds and screen larger areas of the mucosal surface. H. pylori was detected in gastritis using topical staining with acriflavine in a proof-of-principle paper [35] after demonstrating that $\mathrm{H}$. pylori actively incorporates acriflavine in culture. Although H. pylori-diagnosis is standardized and reliably available using established methods, this exemplifies the high resolution of eCLE and was the first example of an in vivo microscopic technique to visualize live bacteria during ongoing endoscopy. A follow-up study showed an accuracy of $93 \%$ to correctly diagnose $H$. pylori-gastritis in vivo [36].

Gastric intestinal metaplasia can be identified with CLE using three criteria, the presence of goblet cells, highly prismatic cells with brush border, and villous transformation of the mucosa (Figure 3(b)). In 267 optical biopsies from 53 patients, intestinal metaplasia was correctly diagnosed with a specificity of $95 \%$ and a sensitivity of $98 \%$. Interobserver agreement for diagnosis during endoscopy was 0.94 [37]. This makes CLE the only reliable endoscopic method to predict intestinal metaplasia. Gastric atrophy was studied with similar results [33].

Differentiation of hyperplastic from adenomatous polyps is often difficult, if only macroscopic criteria are used. Endomicroscopy showed a high accuracy for this differentiation [38], again supporting its use for immediate resection of suspicious lesions. Early trials on gastric cancer have used acriflavine-augmented ex vivo confocal microscopy on biopsy specimens with good accuracy rates [39]. In follow-up studies, disorganized configuration of glands after fluorescein injection were found typical of gastric neoplasia (Figure 3(c)) despite the fact that nuclei were not visible [40]. These results formed the basis for a large trial to establish criteria for gastric cancer. 182 patients were examined in a run-in phase. The evaluation phase included 1786 patients, and eCLE was compared to white light endoscopy for realtime diagnosis of gastric cancer with histopathology as the gold standard. The accuracy of CLE was excellent (99\%) for the in vivo diagnosis of gastric superficial cancer and high grade intraepithelial neoplasia [41]. A video sequence of gastritis, intestinal metaplasia, adenoma, and carcinoma in the same patient has recently been published (Goetz, VJGIE, in press). In Asian countries with a high incidence of gastric neoplasia, endoscopic submucosal dissection (ESD) has become the treatment of choice for gastric adenomas and early gastric cancers. After ESD, R1 at the deep margin is considered an indication for surgery whereas positive lateral margins can be retreated endoscopically. For exact identification of the tumor residues, 24 patients underwent eCLE two weeks after endoscopic resection [42]. CLE identified five patients with indefinite lateral margins that were retreated endoscopically under endomicroscopic guidance. Accuracy of CLE to predict incomplete resection was $92 \%$ in this study. 


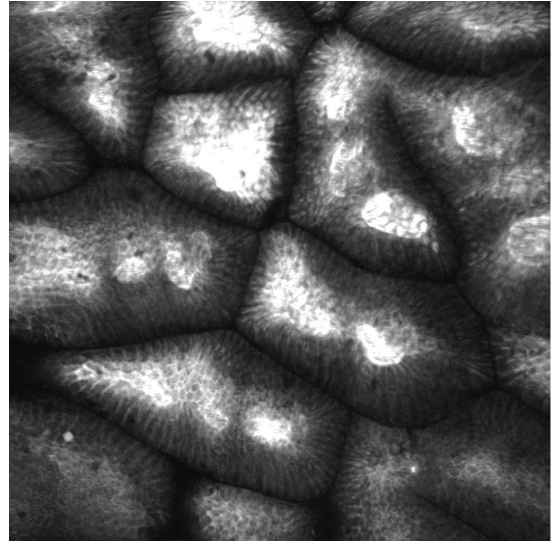

(a)

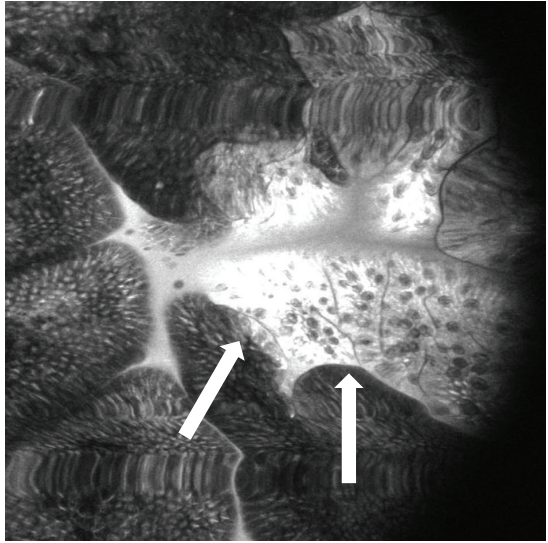

(b)

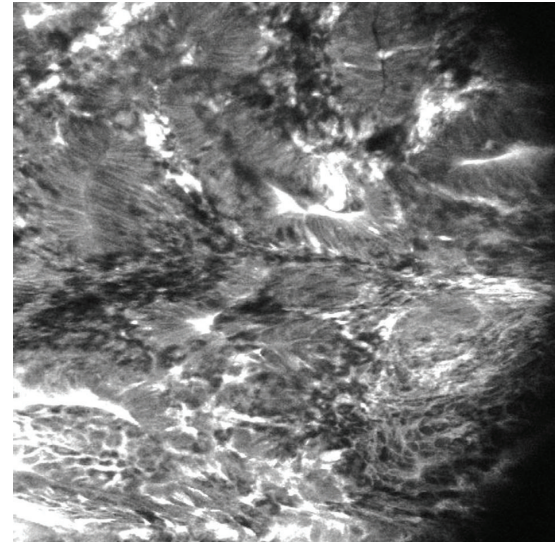

(c)

Figure 3: CLE of the stomach. (a) In the normal antrum, the gastric pits have a branched opening, and superficial sections show a cobblestone appearance of the epithelium that is partially seen on this image. Subepithelial vessels can be seen as bright, often twisted bands. (b) shows a patch of intestinal metaplasia (arrows), easily identifiable by multiple goblet cells (cf. Figure 2(a)) and the brighter color due to a higher pH of cells. (c) In the same patient, a lesion was found that showed typical aspects of gastric cancer with uneven epithelial height of glands, massive extravasation of fluorescein, and cells infiltrating from the epithelium into the lamina propria. Targeted biopsies confirmed gastric cancer. Edge length is $475 \mu \mathrm{m}$.

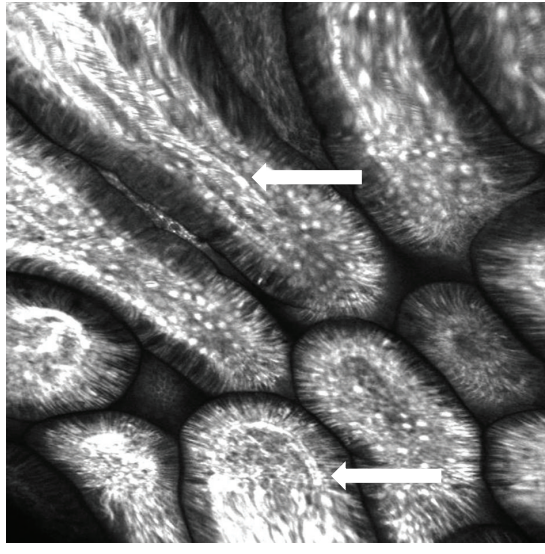

(a)

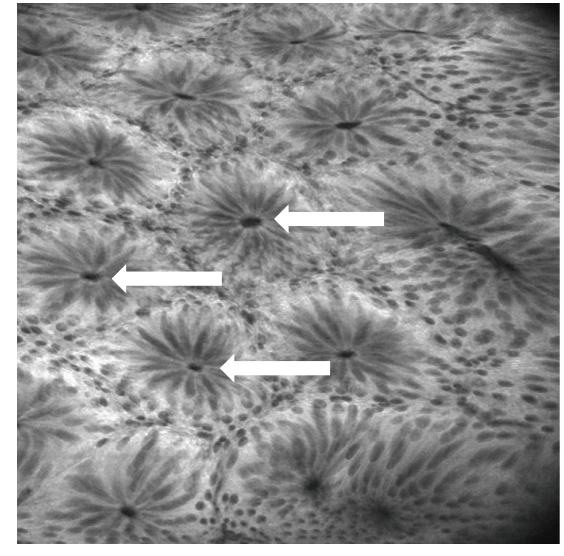

(b)

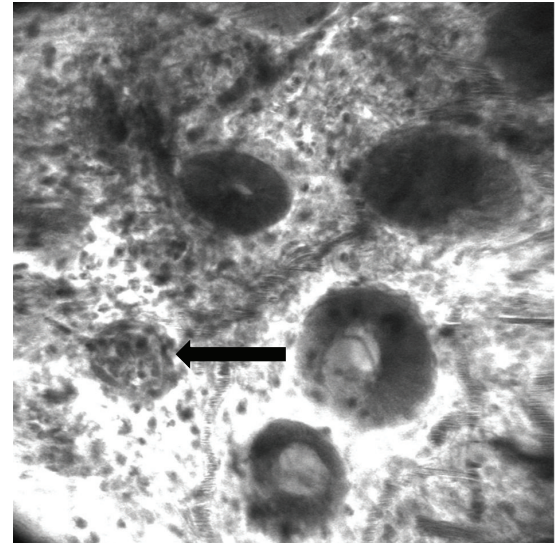

(c)

Figure 4: Terminal ileum, normal and inflamed colon. (a) In the normal terminal ileum, villi are seen with an even epithelial height and hair-pin like vessels (arrows) in the lamina propria. No extravasation of fluorescein is seen. (b) In normal mucosa, mucin rich goblet cells are assorted perpendicular around a crypt opening (arrows), like a slice of orange. (c) In inflammation in ulcerative colitis, vasculature is enriched, fluorescein extravasates, and a crypt abcess (arrow) can be seen. Edge length is $475 \mu \mathrm{m}$.

3.3. Endomicroscopy of the Small Intestine. In the diagnosis of celiac disease, sampling error is common. In a trial on celiac disease, 31 patients were examined by eCLE [43]. Diagnosis of crypt hypertrophy and villous atrophy is easy to obtain with CLE in contrast to quantification of intraepithelial lymphocytes (Marsh I). Still, eCLE showed high accuracy to diagnose celiac disease, and in patients with established celiac disease even a trend towards higher accuracy for CLE versus histopathology was reported due to sampling error for biopsies. These results have been confirmed in followup trials with respect to villous atrophy and intraepithelial lymphocytes, but not crypt hyperplasia [44]. CLE has even been studied in children for the diagnosis of celiac disease. In
19 patients, sensitivity and specificity were $100 \%$ and $80 \%$, respectively [45].

\section{Lower Gastrointestinal Tract}

4.1. Endomicroscopy for Colonic Neoplasia. The first study to evaluate CLE in patients was conducted in screening colonoscopy (Figures 4 and 5) with eCLE to visualize colonic neoplasia and normal colonic mucosa [1]. This resulted in a straightforward applicable classification of colonic changes based on analysis in superficial and deep tissue sections of tissue architecture and vessel structure ("Mainz classification"). This classification system differentiated normal 


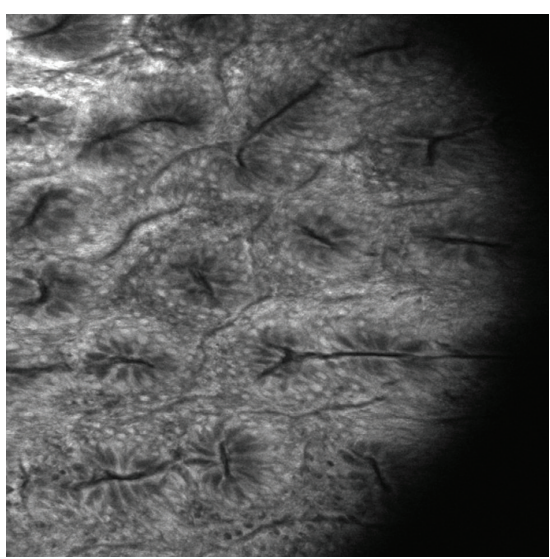

(a)

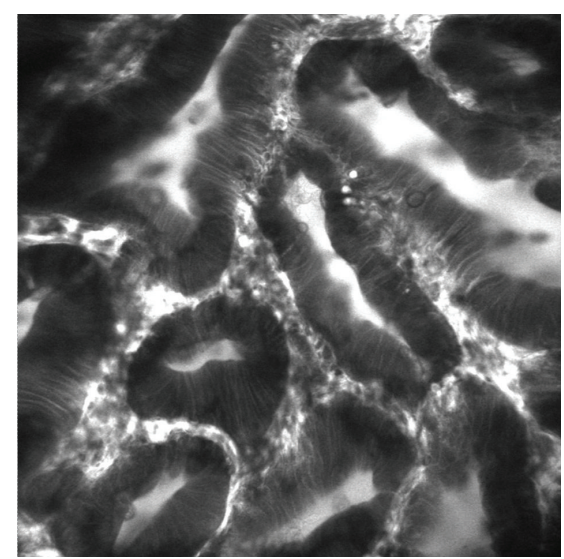

(b)

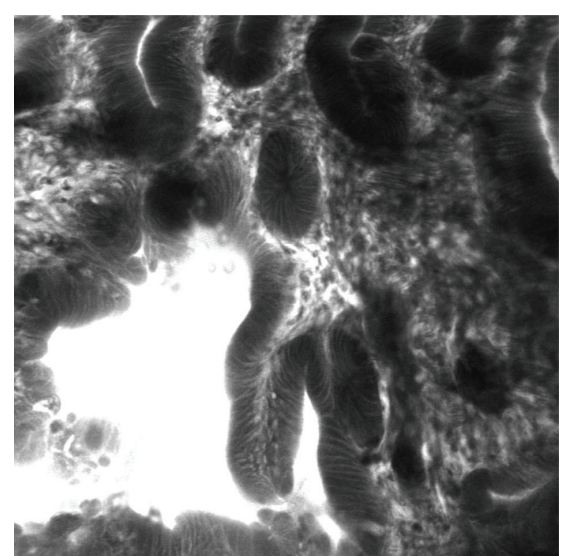

(c)

Figure 5: Colonic polyps. (a) Star-shaped or slit-like crypt openings, normal number of mucinous goblet cells, and a regular crypt size indicate a hyperplastic polyp. (b), (c) Loss of goblet cells, uneven crypt sizes with dark epithelial cells, extravasation of fluorescein into the crypt lumina or the lamina propria indicate adenomas (histology: low-grade colorectal adenomas). Edge length is $475 \mu \mathrm{m}$.

mucosa from regenerative (inflammatory or hyperplastic) from neoplastic alterations with $99 \%$ accuracy. Importantly, the grade of neoplasia was not differentiated due to the lack of visualization of nuclei. However, this landmark study showed for the first time that CLE provides microscopic images during ongoing endoscopy, and that trained endoscopists are able to evaluate such microscopic images on-site. The use of CLE in screening colonoscopy was corroborated by a follow-up study [14]. In order to further grade neoplasia, systemic fluorescein was combined with topical acriflavine, permitting differentiation of high grade versus low grade adenomas [46]. Such visualization of nuclei was also described with cresyl violet in a proof-of-principle study that combined chromoendoscopy and fluorescence imaging with a single dye [15]. A classification system for pCLE has just been published and will undergo further evaluation [47]. A further study compared pCLE for prediction of histology of colonic lesions with virtual chromoendoscopy techniques (NBI, FICE) and found a higher sensitivity for pCLE with similar specificity in classification of colorectal polyps [48]. Such on-site imaging protocols may become even more interesting in the near future, when resect-anddiscard strategies might be incorporated into clinical practice [49].

The infiltration depth of tumors cannot be directly visualized with CLE since imaging plane depth is limited to the mucosa. CLE using near infrared light is able to visualize submucosal structures (own unpublished data), but no appropriate fluorescent contrast agent is available for clinical use at present. However, the submucosa and even muscle layer become accessible after endoscopic (sub-)mucosal resection. Recently, nerves of the Meissner plexus were visualized with CLE [50]. This may offer an opportunity to study and eventually diagnose motility disorders of the colon, an area that has only been incompletely amenable to our current diagnostic tools.
4.2. Endomicroscopy in Colitis. Just as Barrett's esophagus, ulcerative colitis is an excellent example of the application of CLE in clinical practice. Patients with longstanding UC are at increased risk to harbour neoplasia, but neoplasia is difficult to detect by white light endoscopy, and random biopsies have a very low diagnostic yield [51]. On the other hand, panendomicroscopy of the entire colon is not feasible. Therefore CLE has been combined with chromoendoscopy for delivering optimized care to patients under surveillance for longstanding ulcerative colitis. In this setting, chromoendoscopy is used to unmask suspicious regions, and CLE is used for their immediate characterization. eCLE was able to diagnose neoplasia with an accuracy of $98 \%$ in a prospective trial in addition to visualization of inflammatory changes [22] (Figure 4(c)). This is a very high accuracy given the fact that even expert GI histopathologists have lower accuracy values. However again only the presence, but not the grade, of neoplasia was evaluated. A potentially even more important finding was prediction of normal mucosa with an accuracy of over 99\% in optical biopsies (with conventional histopathology as the gold standard). This trial found an approximately 4 -fold increased yield of neoplasia (by the use of chromoendoscopy) with only four targeted biopsies per patient (by the use of CLE), thus basically abrogating the need for random biopsies. Recently, pCLE was found to be able to visualize inflammatory changes in Crohn's disease patients [52].

Microscopic colitis, which encompasses lymphocytic and collagenous colitis, is characterized by normal mucosal findings in conventional endoscopy, but patients suffer from recurrent diarrhea. The microscopic changes often show a patchy distribution within the proximal colon that may be difficult to pick up with unguided random biopsies. In this setting, eCLE has been reported to visualize lymphocytic infiltration in lymphocytic colitis and the subepithelial band in collagenous colitis by taking multiple optical biopsies and by guiding real biopsies microscopically [53-55]. 
In patients with intestinal involvement of graft versus host-disease (GvHD) after bone marrow transplantation, immediate diagnosis and therapy are necessary. In a recent trial, eCLE was able to diagnose and to grade GvHD during ongoing endoscopy with high accuracy based on the presence of inflammation, and epithelial apoptosis could be documented for diagnosis [56]. CLE has not been extensively studied in infectious colitis, but a case report has shown visualization of intestinal spirochaetosis by CLE [57].

\section{Hepatobiliary and Pancreatic Endomicroscopy}

Starting from endoluminal endoscopy, the field of applications for CLE has been rapidly expanding. CLE of the hepatobiliary system has been driven by clinical need to provide optimized diagnosis and by technical developments in the field of endoscopy providing new instruments and novel ways to access intra-abdominal organs.

5.1. Intrabiliary Imaging. Indeterminate biliary strictures are a clinical dilemma. Conventional methods to evaluate strictures include brush cytology, with an excellent specificity, but sensitivities well below $50 \%$. Outcome is optimized when combined with other clinical imaging modalities including cholangioscopy-guided biopsies [58] but results are still much less reliable than in other endoscopic fields. The availability of pCLE has permitted transpapillary intrabiliary introduction of microscopy probes via a duodenoscope after systemic administration of fluorescein. The first study from an expert center to evaluate biliary strictures has found a sensitivity of $83 \%$ for pCLE as compared to $50 \%$ for histology. Although image quality was lower in the biliary tree than in mucosal imaging in the GI tract, the presence of neoplasia was suggested by irregular, dilated vessels [59]. Lower accuracy rates were reported from a follow-up trial [60]. The reason for a somewhat lower image quality with bile duct imaging may be partially related to the different microarchitecture of the biliary epithelium, but also to compromises in the resolution due to access with an ultrathin probe. Recently, a pCLE probe routinely used for gastric imaging was introduced into the bile duct and showed higher image quality compared to the biliary probe type [61].

In order to standardize CLE criteria for diagnosis of neoplasia within the bile duct, the "Miami classification" was published [62] and recently supplemented by a set of pCLE criteria for indeterminate biliary and pancreatic strictures derived from a large data set by expert blinded reviewing of pCLE video sequences [63]. Criteria of malignancy were thick white bands $(>20 \mu \mathrm{m})$, thick dark bands $(>40 \mu \mathrm{m})$, or dark clumps or epithelial structures. The combination of more than two criteria provided sensitivity, specificity, positive predictive value, and negative predictive value of $97 \%, 33 \%, 80 \%$, and $80 \%$, respectively, compared with $48 \%$, $100 \%, 100 \%$, and $41 \%$ for standard tissue sampling. The differences with regard to sensitivity and specificity indicate that PCLE may be a valuable adjunct to brush cytology in indeterminate strictures.
The histopathologic correlate of the pCLE findings has not been finally elucidated, since multiple tissue samples are difficult to obtain from the bile duct. Current interpretations suggest that white bands most probably correspond to blood vessels (filled with fluorescein), black bands may be the correlate of lymphatic vessels, and black clumps of neoplastic epithelial structures. Another difficult clinical problem, the presence of biliary strictures in primary sclerosing cholangitis, has not yet been addressed in studies.

Recently, pCLE has also been evaluated for imaging in the pancreatic duct. Similar morphologic criteria as in biliary strictures have been described for pancreatic strictures in a pilot study [63]. Since many pancreatic anomalies are not directly accessible via the main duct, pCLE has been combined with endoscopic ultrasound (EUS). Here, EUS provides macroscopic targeting with a puncture needle. A pCLE probe is then advanced through the needle to provide imaging within a pancreatic (cystic) lesion [64]. Such imaging may be an interesting alternative in situations where technically difficult tissue sampling could benefit from microscopic targeting of the biopsy site.

5.2. Intra-Abdominal Endomicroscopy. Natural orifice transluminal endoscopic surgery (NOTES) has prompted the exploration of CLE within the abdominal cavity. Two earlier animal trials showing the feasibility of intra-abdominal imaging $[65,66]$ have not (yet) been followed by subsequent clinical applications.

Two studies have been published for confocal laparoscopy in humans. In the first trial, a rigid confocal prototype probe of $6 \mathrm{~mm}$ outer diameter was based on the FIVE1-design with optical properties similar to eCLE and covered with a sterile metal sheath to allow for sterile intraabdominal instrumentation [67]. Under minilaparoscopic guidance and conscious sedation $[68,69]$ the probe was positioned onto the liver, and imaging performed after fluorescein injection. Although basic features of the liver microarchitecture could be visualized, images could not provide sufficient details to allow exact diagnosis in liver diseases, probably due to the fact that the human liver capsule did not allow sufficient penetration of blue laser light to the parenchyma. Therefore in a follow-up trial, a similar prototype probe was equipped with a near infrared light source of $780 \mathrm{~nm}$ [70], and fluorescein was changed to indocyanine green for tissue contrast [71]. In this study, images of the human liver revealed details (including nuclei) of the parenchyma in vivo comparable to standard histology in some aspects. Accuracy of near infrared confocal laparoscopy to predict the presence of steatosis and fibrosis was $81 \%$ and $90 \%$, respectively. However, nonparenchymal aspects were only indirectly visualized.

Although for both intrabiliary and intra-abdominal/liver confocal microscopy applications first clinical trials have shown feasibility in human trials, images from these indications do not yet quite compete with image quality from GI mucosal imaging, and more trials are awaited to optimize imaging. Feasibility studies to evaluate confocal imaging for intraoperative screening are under way [72]. 


\section{Expanding the Field of Indication in Medicine}

The basic principles of intravital endomicroscopy are not limited to the field of gastroenterology. Every organ that can be accessed in diagnostic or therapeutic medicine may be subject to CLE examination. In fact, one of the first human trials has been conducted in dermatology with a scanner later used for eCLE [73, 74]. After first studies using pCLE [75] or eCLE/FIVE1 [76] for imaging of tissue excised from the urinary bladder, pCLE has been evaluated for onsite diagnosis in urology [77]. eCLE has been studied in imaging of neoplasias of the oral cavity [78] and-using a rigid scanner (used as a prototype for confocal laparoscopy, see above)-for intraoperative imaging of neoplasia in the larynx [79]. Pilot trials have evaluated rigid CLE for imaging of cervical intraepithelial neoplasia during colposcopy, using acriflavine [80]. Imaging to guide resection in neurosurgery is currently under evaluation [81-83].

\section{Functional and Molecular Imaging with Endomicroscopy}

7.1. Animal Studies. While CLE has been initially considered to provide in vivo histology, this is not entirely true. Apart from many aspects of conventional histology that are not rendered by CLE, CLE also offers the unique option to visualize microscopic details of tissue in the natural micromilieu without disrupting tissue integrity and is therefore far less prone to artifacts by sampling, staining, or cutting. Besides, in vivo imaging is able to add a time axis to microscopy, thereby permitting dynamic (rather than static) imaging [84]. The confocal scanner that was later integrated into eCLE endoscopes has been available for laboratory use for years (FIVE1, Optiscan) [85-87], and also the probe based system can be used in the laboratory. Animal studies do not face the limitations and safety constraints of human trials so that many of the contrast agents used for bench top laser scanning microscopy can be used in vivo [16]. Initial studies have evaluated intravenous fluorescein, that was later rapidly transferred into human applications, but also used intravenous acriflavine. The latter, by staining nuclei and cytoplasm, provides an intravital image that is comparable to hematoxylin and eosin staining [88]. By the high resolution of the handheld eCLE scanner, subcellular details of (patho)physiologic processes can be unraveled in vivo. For example, it has been demonstrated in a murine model of hepatocyte apoptosis, where single cells have been observed for up to 6 hours within the intact liver in a live mouse, that cells that are to undergo apoptosis show swelling of the cytoplasm rather than shrinkage as a first sign in the initial phase of apoptosis [91]. Such procedural, dynamic imaging in the whole organ with intact perfusion and micromilieu cannot be repeated ex vivo.

The injection of FITC-labeled dextrans of different molecular weights offers the opportunity to study leakage of blood compounds in different inflammatory states $[89,90]$ or in diseases of dysregulated apoptosis such as liver failure where capillary leakage is believed to be an early event [91]. Leakage of labeled Lycopersicon esculentum-Lectin that was used to label vessel walls in vivo was found helpful to identify metastatic foci of few cells in the liver [16]. The combination of different dyes allowed comprehensive imaging of tissue and vessel function against a background of tissue morphologic staining $[89,92]$, although multiwavelength imaging and overlay of different excitation and emission lines is not (yet) possible. The combination of animal imaging devices and devices intended for human use has therefore opened the door for rapid translation from bench to bedside.

7.2. Functional Imaging. Translational imaging has been shown to identify gaps in the epithelial layer of healthy gut mucosa. Such gap formation seems to be part of normal epithelial regeneration, and gaps smaller or just the size of a single epithelial cell seem to be present in a large number but sealed by a yet unidentified substance to preserve epithelial barrier function [93]. In a further step, the characteristics of such gaps have been defined in healthy mice in vivo and after the induction of colitis, and subsequently have been visualized with eCLE in healthy patients [94]. In subsequent studies it has been found that gap density is increased in patients with Crohn's Disease. Whereas control patients only showed 18 gaps per 1000 epithelial cells, 117 gaps were found in patients with inflammatory bowel disease using pCLE $[95,96]$. Such gaps coincide with increased bacterial translocation in patients with inflammatory bowel diseases. Similar to Helicobacter pylori in the stomach [35], bacteria were also identified using fluorescein in the human colon as tiny white dots within the lamina propria and were confirmed by staining of microscopically targeted biopsies. Intramucosal bacteria were significantly more frequent in patients with inflammatory bowel diseases than in healthy controls [97]. Again, imaging in intact tissue offers the great advantage of not submitting disrupted tissue (biopsies) to potential cross contamination during sampling by forceps, bowel contents or during processing. Exposition of intestinal mucosa to bacteria induces increased cell shedding. To further delineate the function of epithelial gaps in inflammatory bowel diseases, gaps without transepithelial leakage after systemic fluorescein administration have been differentiated from gaps with leakage and microerosions in patients with endoscopic and histologic remission. Those latter gaps seem to confer an impairment of the mucosal barrier function, since patients with gaps and leakage had an approximately fourfold increased risk to relapse over the course of 12 months [98]. These trials of translational imaging suggest gaps as a microarchitectural morphological correlate of impaired epithelial barrier function in inflammatory bowel diseases, and mucosal healing on functional level as a predictor of sustained remission.

Another functional aspect in tissue imaging that relies on in vivo imaging and can be investigated by the use of CLE is tissue perfusion [99]. Increased vessel leakiness indicated by extravasation of fluorescein can be seen regularly in ulcerative colitis [22] and colonic neoplasia [1]. Changes of the capillary network density have helped to identify 
neoplasia in Barrett's esophagus [24]. Taken together, these studies illustrate the ability of CLE to visualize physiologic events in vivo on a microscopic level. They have the potential to induce a paradigm shift in our understanding of gastrointestinal diseases.

7.3. Molecular Imaging. All the above-mentioned CLE applications have used fairly unspecific staining protocols, mostly fluorescein-based. Similar to bench top microscopy, CLE signal can be read out after administration of labeled exogenous molecular probes such as peptides, antibodies, or probes with tumor-specific activation [100, 101]. At present, molecular imaging in gastrointestinal endoscopy aims at two major applications (Goetz GIE 2012). The first is enhanced detection and lower miss rate of neoplasias within the gastrointestinal lumen. This is mostly being studied by using wide-field endoscopes detecting induced fluorescence. The second application of molecular imaging is improved characterization of lesions that can even be used for prediction of response to molecular targeted therapy.

For a first trial in humans using pCLE, a heptapeptide had been derived from a phage library that showed preferential binding to human colorectal adenoma cells. After FITClabeling, molecular imaging of adenoma was demonstrated during colonoscopy after topical administration during endoscopy [102]. In a trial using a human-murine xenograft model, colorectal cancer cells were specifically visualized with eCLE after injection of labeled antibodies targeting human epidermal growth factor receptor (EGFR) [103]. After topical application, even EGFR on excised human cancer specimens could be quantified. A follow-up study identified vascular endothelial growth factor (VEGF) as a potential target for molecular CLE [104]. Such imaging of receptors or ligands overexpressed in tumors is integrated into therapy protocols of neuroendocrine tumors, for example, by scintigraphy. With CLE, imaging of somatostatin receptor has been achieved in murine models of neuroendocrine tumors $[16,105]$. This concept of in vivo tumor imaging has been supplemented by using not just diagnostic, but therapeutic antibodies. Recently, it has been demonstrated that labeled Cetuximab, a therapeutic agent targeting human EGFR, can be used to visualize gastric cancer cells with CLE in a xenograft model [106]. Such use of in vivo therapeutic antibody labeling has been connected to prediction of response, where high fluorescence intensity as quantified by molecular targeted CLE was correlated to slower tumor growth and better thriving and overall survival in a mouse model of human colorectal cancer [107]. This study exemplifies the role of CLE in personalizing treatment in inflammatory and neoplastic diseases. But CLE also has the option to visualize binding patterns on a single cell level in vivo [106] with the potential to image drug-cellular interaction in vivo.

\section{Summary}

In summary, confocal laser endomicroscopy is a novel tool in the endoscopist's armamentarium that is fundamentally different in its ability to visualize the mucosa on a subcellular level. In addition to providing on site histologic information at real-time, CLE allows "smart," targeted biopsies and is able to guide endoscopic interventions. But CLE is also translational in its approach and permits functional imaging that significantly impacts on our understanding of gastrointestinal diseases. Molecular imaging with CLE allows detection and characterization of lesions and may even be used for prediction of response to targeted therapy.

\section{Abbreviations}

CLE: Confocal laser endomicroscopy

EGFR: Epidermal growth factor receptor

EUS: Endoscopic ultrasound

GvHD: Graft versus host disease

IBD: Inflammatory bowel disease

VEGF: Vascular endothelial growth factor.

\section{References}

[1] R. Kiesslich, J. Burg, M. Vieth et al., "Confocal laser endoscopy for diagnosing intraepithelial neoplasias and colorectal cancer in vivo," Gastroenterology, vol. 127, no. 3, pp. 706-713, 2004.

[2] M. Goetz, A. Watson, and R. Kiesslich, "Confocal laser endomicroscopy in gastrointestinal diseases," Journal of Biophotonics, vol. 4, no. 7-8, pp. 498-508, 2011.

[3] A. Hoffman, M. Goetz, M. Vieth, P. R. Galle, M. F. Neurath, and R. Klesslich, "Confocal laser endomicroscopy technical status and current indications," Endoscopy, vol. 38, no. 12, pp. 1275-1283, 2006.

[4] R. Kiesslich, M. Goetz, and M. F. Neurath, "Confocal Laser Endomicroscopy for Gastrointestinal Diseases," Gastrointestinal Endoscopy Clinics of North America, vol. 18, no. 3, pp. 451-466, 2008.

[5] R. Kiesslich, M. Goetz, M. Vieth, P. R. Galle, and M. F. Neurath, "Technology insight: confocal laser endoscopy for in vivo diagnosis of colorectal cancer," Nature Clinical Practice Oncology, vol. 4, no. 8, pp. 480-490, 2007.

[6] G. D. De Palma, M. B. Wallace, and M. Giovannini, "Confocal laser endomicroscopy," Gastroenterology Research and Practice, vol. 2012, Article ID 216209, 2 pages, 2012.

[7] M. B. Wallace and P. Fockens, "Probe-based confocal laser endomicroscopy," Gastroenterology, vol. 136, no. 5, pp. 15091513, 2009.

[8] V. Becker, T. Vercauteren, C. H. von Weyhern, C. Prinz, R. M. Schmid, and A. Meining, "High-resolution miniprobe-based confocal microscopy in combination with video mosaicing (with video)," Gastrointestinal Endoscopy, vol. 66, no. 5, pp. 1001-1007, 2007.

[9] A. Meining, "Confocal endomicroscopy," Gastrointestinal Endoscopy Clinics of North America, vol. 19, no. 4, pp. 629635, 2009.

[10] R. S. Kwon, L. M. Wong Kee Song, D. G. Adler et al., "Endocytoscopy," Gastrointestinal Endoscopy, vol. 70, no. 4, pp. 610-613, 2009.

[11] K. Sasajima, S. E. Kudo, H. Inoue et al., "Real-time in vivo virtual histology of colorectal lesions when using the endocytoscopy system," Gastrointestinal Endoscopy, vol. 63, no. 7, pp. 1010-1017, 2006. 
[12] S. E. Kudo, K. Wakamura, N. Ikehara, Y. Mori, H. Inoue, and S. Hamatani, "Diagnosis of colorectal lesions with a novel endocytoscopic classification-a pilot study," Endoscopy, vol. 43, pp. 869-875, 2011.

[13] M. B. Wallace, A. Meining, M. I. Canto et al., "The safety of intravenous fluorescein for confocal laser endomicroscopy in the gastrointestinal tract," Alimentary Pharmacology and Therapeutics, vol. 31, no. 5, pp. 548-552, 2010.

[14] A. L. Polglase, W. J. McLaren, S. A. Skinner, R. Kiesslich, M. F. Neurath, and P. M. Delaney, "A fluorescence confocal endomicroscope for in vivo microscopy of the upper- and the lower-GI tract," Gastrointestinal Endoscopy, vol. 62, no. 5, pp. 686-695, 2005.

[15] M. Goetz, T. Toermer, M. Vieth et al., "Simultaneous confocal laser endomicroscopy and chromoendoscopy with topical cresyl violet," Gastrointestinal Endoscopy, vol. 70, no. 5, pp. 959-968, 2009.

[16] M. Goetz, C. Footner, E. Schirrmacher et al., "In-vivo confocal real-time mini-microscopy in animal models of human inflammatory and neoplastic diseases," Endoscopy, vol. 39, no. 4, pp. 350-356, 2007.

[17] K. B. Dunbar, E. A. Montgomery, and M. I. Canto, "The learning curve of in vivo confocal laser endomicroscopy for prediction of Barrett's esophagus," Gastroenterology, vol. 134, supplement 1, no. 4, pp. A-62-A-63, 2008.

[18] http://www.endomicroscopy.org/learningcenter/.

[19] R. Kiesslich, P. R. Galle, and M. F. Neurath, Eds., Atlas of Endomicroscopy, Springer Medizin, Heidelberg, Germany, 2008.

[20] A. M. Buchner, V. Gomez, M. G. Heckman et al., "The learning curve of in vivo probe-based confocal laser endomicroscopy for prediction of colorectal neoplasia," Gastrointestinal Endoscopy, vol. 73, no. 3, pp. 556-560, 2011.

[21] K. B. Dunbar, P. Okolo, E. Montgomery, and M. I. Canto, "Confocal laser endomicroscopy in Barrett's esophagus and endoscopically inapparent Barrett's neoplasia: a prospective, randomized, double-blind, controlled, crossover trial," Gastrointestinal Endoscopy, vol. 70, no. 4, pp. 645-654, 2009.

[22] R. Kiesslich, M. Goetz, K. Lammersdorf et al., "Chromoscopy-guided endomicroscopy increases the diagnostic yield of intraepithelial neoplasia in ulcerative colitis," Gastroenterology, vol. 132, no. 3, pp. 874-882, 2007.

[23] K. Y. Lin, M. Maricevich, N. Bardeesy, R. Weissleder, and U. Mahmood, "In vivo quantitative microvasculature phenotype imaging of healthy and malignant tissues using a fiberoptic confocal laser microprobe," Translational Oncology, vol. 1, pp. 84-94, 2008.

[24] V. Becker, M. Vieth, M. Bajbouj, R. M. Schmid, and A. Meining, "Confocal laser scanning fluorescence microscopy for in vivo determination of microvessel density in Barrett's esophagus," Endoscopy, vol. 40, no. 11, pp. 888-891, 2008.

[25] R. Tous, J. Delgado, T. Zinkl et al., "The anatomy of an optical biopsy semantic retrieval system," IEEE MultiMedia, vol. 19, pp. 16-27, 2012.

[26] B. Andre, T. Vercauteren, A. M. Buchner, M. W. Shahid, M. B. Wallace, and N. Ayache, "An image retrieval approach to setup difficulty levels in training systems for endomicroscopy diagnosis," Medical Image Computing and Computer-Assisted Intervention, vol. 13, pp. 480-487, 2010.

[27] O. Pech, T. Rabenstein, H. Manner et al., "Confocal laser endomicroscopy for in vivo diagnosis of early squamous cell carcinoma in the esophagus," Clinical Gastroenterology and Hepatology, vol. 6, no. 1, pp. 89-94, 2008.
[28] H. Liu, Y. Q. Li, T. Yu et al., "Confocal laser endomicroscopy for superficial esophageal squamous cell carcinoma," Endoscopy, vol. 41, no. 2, pp. 99-106, 2009.

[29] R. Kiesslich, L. Gossner, M. Goetz et al., "In vivo histology of Barrett's esophagus and associated neoplasia by confocal laser endomicroscopy," Clinical Gastroenterology and Hepatology, vol. 4, no. 8, pp. 979-987, 2006.

[30] H. Pohl, T. Rösch, M. Vieth et al., "Miniprobe confocal laser microscopy for the detection of invisible neoplasia in patients with Barrett's oesophagus," Gut, vol. 57, no. 12, pp. 1648 1653, 2008.

[31] M. Bajbouj, M. Vieth, T. Rösch et al., "Probe-based confocal laser endomicroscopy compared with standard fourquadrant biopsy for evaluation of neoplasia in Barretts esophagus," Endoscopy, vol. 42, no. 6, pp. 435-440, 2010.

[32] C. L. Chu, Y. B. Zhen, G. P. Lv et al., "Microalterations of esophagus in patients with non-erosive reflux disease: in-vivo diagnosis by confocal laser endomicroscopy and its relationship with gastroesophageal reflux," The American Journal of Gastroenterology, vol. 107, pp. 864-874, 2012.

[33] J. N. Zhang, Y. Q. Li, Y. A. Zhao et al., "Classification of gastric pit patterns by confocal endomicroscopy," Gastrointestinal Endoscopy, vol. 67, no. 6, pp. 843-853, 2008.

[34] P. Wang, R. Ji, T. Yu et al., "Classification of histological severity of Helicobacter pylori-associated gastritis by confocal laser endomicroscopy," World Journal of Gastroenterology, vol. 16, no. 41, pp. 5203-5210, 2010.

[35] R. Kiesslich, M. Goetz, J. Burg et al., "Diagnosing Helicobacter pylori in vivo by confocal laser endoscopy," Gastroenterology, vol. 128, no. 7, pp. 2119-2123, 2005.

[36] R. Ji, Y. Q. Li, X. M. Gu, T. Yu, X. L. Zuo, and C. J. Zhou, "Confocal laser endomicroscopy for diagnosis of Helicobacter pylori infection: a prospective study," Journal of Gastroenterology and Hepatology, vol. 25, no. 4, pp. 700-705, 2010.

[37] Y. T. Guo, Y. Q. Li, T. Yu et al., "Diagnosis of gastric intestinal metaplasia with confocal laser endomicroscopy in vivo: a prospective study," Endoscopy, vol. 40, no. 7, pp. 547-553, 2008.

[38] W. B. Li, X. L. Zuo, F. Zuo et al., "Characterization and identification of gastric hyperplastic polyps and adenomas by confocal laser endomicroscopy," Surgical Endoscopy and Other Interventional Techniques, vol. 24, no. 3, pp. 517-524, 2010.

[39] K. G. Yeoh, M. Salto-Tellez, and C. J. Khor, Confocal Laser Endoscopy Is Useful for In-Vivo Rapid diagnosIs of Gastric Neoplasia and Preneoplasia, Digestive Disease Weak, Chicago, Ill, USA, 2005.

[40] S. Kitakabe, Y. Niwa, and Y. Hirooka, Confocal Laser Endoscopy for the Diagnosis of Gastric Diseases In Vivo, Digestive Disease Weak, Chicago, Ill, USA, 2005.

[41] W. B. Li, X. L. Zuo, C. Q. Li et al., "Diagnostic value of confocal laser endomicroscopy for gastric superficial cancerous lesions," Gut, vol. 60, no. 3, pp. 299-306, 2011.

[42] R. Ji, X. L. Zuo, C. Q. Li, C. J. Zhou, and Y. Q. Li, “Confocal endomicroscopy for in vivo prediction of completeness after endoscopic mucosal resection," Surgical Endoscopy, vol. 25, no. 6, pp. 1933-1938, 2010.

[43] R. W. L. Leong, N. Q. Nguyen, C. G. Meredith et al., "In vivo confocal endomicroscopy in the diagnosis and evaluation of celiac disease," Gastroenterology, vol. 135, no. 6, pp. 18701876, 2008. 
[44] U. Günther, S. Daum, F. Heller et al., "Diagnostic value of confocal endomicroscopy in celiac disease," Endoscopy, vol. 42, no. 3, pp. 197-202, 2010.

[45] K. Venkatesh, A. Abou-Taleb, M. Cohen et al., "Role of confocal endomicroscopy in the diagnosis of celiac disease," Journal of Pediatric Gastroenterology and Nutrition, vol. 51, no. 3, pp. 274-279, 2010.

[46] S. Sanduleanu, A. Driessen, E. Gomez-Garcia, W. Hameeteman, A. de Bruïne, and A. Masclee, "In vivo diagnosis and classification of colorectal neoplasia by chromoendoscopyguided confocal laser endomicroscopy," Clinical Gastroenterology and Hepatology, vol. 8, no. 4, pp. 371-378, 2010.

[47] T. Kuiper, F. J. van den Broek, S. van Eeden et al., "New classification for probe-based confocal laser endomicroscopy in the colon," Endoscopy, vol. 43, pp. 1076-1081, 2011.

[48] A. M. Buchner, M. W. Shahid, M. G. Heckman et al., "Comparison of probe-based confocal laser endomicroscopy with virtual chromoendoscopy for classification of colon polyps," Gastroenterology, vol. 138, no. 3, pp. 834-842, 2010.

[49] D. K. Rex, C. Kahi, M. O’Brien et al., “The American Society for Gastrointestinal Endoscopy PIVI (Preservation and Incorporation of Valuable Endoscopic Innovations) on realtime endoscopic assessment of the histology of diminutive colorectal polyps," Gastrointestinal Endoscopy, vol. 73, no. 3, pp. 419-422, 2011.

[50] K. Sumiyama, R. Kiesslich, T. R. Ohya, M. Goetz, and H. Tajiri, "In vivo imaging of enteric neuronal networks in humans using confocal laser endomicroscopy," Gastroenterology, vol. 143, pp. 1152-1153, 2012.

[51] F. J. C. van den Broek, P. C. F. Stokkers, J. B. Reitsma et al., "Random biopsies taken during colonoscopic surveillance of patients with longstanding ulcerative colitis: low yield and absence of clinical consequences," American Journal of Gastroenterology. In press.

[52] H. Neumann, M. Vieth, R. Atreya et al., "Assessment of Crohn's disease activity by confocal laser endomicroscopy," Inflammatory Bowel Diseases, vol. 18, no. 12, pp. 2261-2269, 2012.

[53] R. Kiesslich, A. Hoffman, M. Goetz et al., "In vivo diagnosis of collagenous colitis by confocal endomicroscopy," Gut, vol. 55, no. 4, pp. 591-592, 2006.

[54] A. Zambelli, V. Villanacci, E. Buscarini, G. Bassotti, and L. Albarello, "Collagenous colitis: a case series with confocal laser microscopy and histology correlation," Endoscopy, vol. 40, no. 7, pp. 606-608, 2008.

[55] A. Meining, S. Schwendy, V. Becker, R. M. Schmid, and C. Prinz, "In vivo histopathology of lymphocytic colitis," Gastrointestinal Endoscopy, vol. 66, no. 2, pp. 398-400, 2007.

[56] C. Bojarski, U. Günther, K. Rieger et al., "In vivo diagnosis of acute intestinal graft-versus-host disease by confocal endomicroscopy," Endoscopy, vol. 41, no. 5, pp. 433-438, 2009.

[57] U. Günther, H. J. Epple, F. Heller et al., "In vivo diagnosis of intestinal spirochaetosis by confocal endomicroscopy," Gut, vol. 57, no. 9, pp. 1331-1333, 2008.

[58] T. Rösch, K. Hofrichter, E. Frimberger et al., "ERCP or EUS for tissue diagnosis of biliary strictures? A prospective comparative study," Gastrointestinal Endoscopy, vol. 60, no. 3, pp. 390-396, 2004.

[59] A. Meining, E. Frimberger, V. Becker et al., "Detection of cholangiocarcinoma in vivo using miniprobe-based confocal fluorescence microscopy," Clinical Gastroenterology and Hepatology, vol. 6, no. 9, pp. 1057-1060, 2008.

[60] C. S. Loeser, M. E. Robert, A. Mennone, M. H. Nathanson, and P. Jamidar, "Confocal endomicroscopic examination of malignant biliary strictures and histologic correlation with lymphatics," Journal of Clinical Gastroenterology, vol. 45, no. 3, pp. 246-252, 2011.

[61] F. K. Shieh, H. Drumm, M. H. Nathanson, and P. A. Jamidar, "High-definition confocal endomicroscopy of the common bile duct," Journal of Clinical Gastroenterology, vol. 46, pp. 401-406, 2012.

[62] M. Wallace, G. Y. Lauwers, Y. Chen et al., "Miami classification for probe-based confocal laser endomicroscopy," Endoscopy, vol. 43, pp. 882-891, 2011.

[63] A. Meining, R. J. Shah, A. Slivka et al., "Classification of probe-based confocal laser endomicroscopy findings in pancreaticobiliary strictures," Endoscopy, vol. 44, pp. 251257, 2012.

[64] V. J. Konda, H. R. Aslanian, M. B. Wallace, U. D. Siddiqui, J. Hart, and I. Waxman, "First assessment of needle-based confocal laser endomicroscopy during EUS-FNA procedures of the pancreas (with videos)," Gastrointestinal Endoscopy, vol. 74, no. 5, pp. 1049-1060, 2011.

[65] S. von Delius, H. Feussner, D. Wilhelm et al., "Transgastric in vivo histology in the peritoneal cavity using miniprobebased confocal fluorescence microscopy in an acute porcine model," Endoscopy, vol. 39, no. 5, pp. 407-411, 2007.

[66] S. V. Kantsevoy, R. Kiesslich, X. Dray et al., "Translumenal intraperitoneal confocal laser endomicroscopy: a pilot study in a porcine model," Gastrointestinal Endoscopy, vol. 67, p. AB117, 2008.

[67] M. Goetz, R. Klesslich, H. P. Dienes et al., "In vivo confocal laser endomicroscopy of the human liver: a novel method for assessing liver microarchitecture in real time," Endoscopy, vol. 40, no. 7, pp. 554-562, 2008.

[68] U. Denzer, A. Arnoldy, S. Kanzler, P. R. Galle, H. P. Dienes, and A. W. Lohse, "Prospective randomized comparison of minilaparoscopy and percutaneous liver biopsy: diagnosis of cirrhosis and complications," Journal of Clinical Gastroenterology, vol. 41, no. 1, pp. 103-110, 2007.

[69] A. Hoffman, F. Rahman, S. Prengel et al., "Mini-laparoscopy in the endoscopy unit: safety and outcomes in over one thousand patients," The World Journal of Gastrointestinal Endoscopy, vol. 3, pp. 6-10, 2011.

[70] M. Goetz, I. Deris, M. Vieth et al., "Near-infrared confocal imaging during mini-laparoscopy: a novel rigid endomicroscope with increased imaging plane depth," Journal of Hepatology, vol. 53, no. 1, pp. 84-90, 2010.

[71] T. Ishizawa, S. Tamura, K. Masuda et al., "Intraoperative fluorescent cholangiography using indocyanine green: a biliary road map for safe surgery," Journal of the American College of Surgeons, vol. 208, no. 1, pp. e1-e4, 2009.

[72] N. Q. Nguyen, A. V. Biankin, R. W. Leong et al., "Real time intraoperative confocal laser microscopy-guided surgery," Annals of Surgery, vol. 249, pp. 735-737, 2009.

[73] L. D. Swindle, S. G. Thomas, M. Freeman, and P. M. Delaney, "View of normal human skin in vivo as observed using fluorescent fiber-optic confocal microscopic imaging," Journal of Investigative Dermatology, vol. 121, no. 4, pp. 706712, 2003.

[74] P. Anikijenko, L. T. Vo, E. R. Murr et al., "In vivo detection of small subsurface melanomas in athymic mice using noninvasive fiber optic confocal imaging," Journal of Investigative Dermatology, vol. 117, no. 6, pp. 1442-1448, 2001. 
[75] G. A. Sonn, K. E. Mach, K. Jensen et al., "Fibered confocal microscopy of bladder tumors: an ex vivo study," Journal of Endourology, vol. 23, no. 2, pp. 197-201, 2009.

[76] C. Wiesner, W. Jäger, A. Salzer et al., "Confocal laser endomicroscopy for the diagnosis of urothelial bladder neoplasia: a technology of the future?" British Journal of Urology International, vol. 107, no. 3, pp. 399-403, 2011.

[77] G. A. Sonn, S. N. E. Jones, T. V. Tarin et al., "Optical biopsy of human bladder neoplasia with in vivo confocal laser endomicroscopy," Journal of Urology, vol. 182, no. 4, pp. 1299-1305, 2009.

[78] B. R. Haxel, M. Goetz, R. Kiesslich, and J. Gosepath, "Confocal endomicroscopy: a novel application for imaging of oral and oropharyngeal mucosa in human," European Archives of Oto-Rhino-Laryngology, vol. 267, no. 3, pp. 443448, 2010.

[79] B. Pogorzelski, U. Hanenkamp, M. Goetz, R. Kiesslich, and J. Gosepath, "Systematic intraoperative application of confocal endomicroscopy for early detection and resection of squamous cell carcinoma of the head and neck: a preliminary report," Archives of Otolaryngology-Head \& Neck Surgery, vol. 138, pp. 404-411, 2012.

[80] J. Tan, M. A. Quinn, J. M. Pyman, P. M. Delaney, and W. J. McLaren, "Detection of cervical intraepithelial neoplasia in vivo using confocal endomicroscopy," British Journal of Obstetrics and Gynaecology, vol. 116, no. 12, pp. 1663-1670, 2009.

[81] S. Foersch, A. Heimann, A. Ayyad et al., "Confocal laser endomicroscopy for diagnosis and histomorphologic imaging of brain tumors in vivo," PLoS One, vol. 7, no. 7, article e41760, 2012.

[82] J. Eschbacher, N. L. Martirosyan, P. Nakaji et al., "In vivo intraoperative confocal microscopy for real-time histopathological imaging of brain tumors," Journal of Neurosurgery, vol. 116, pp. 854-860, 2012.

[83] N. L. Martirosyan, D. D. Cavalcanti, J. M. Eschbacher et al., "Use of in vivo near-infrared laser confocal endomicroscopy with indocyanine green to detect the boundary of infiltrative tumor," Journal of Neurosurgery, vol. 115, pp. 1131-1138, 2011.

[84] M. Goetz and R. Kiesslich, "Advances of endomicroscopy for gastrointestinal physiology and diseases," American Journal of Physiology-Gastrointestinal and Liver Physiology, vol. 298, no. 6, pp. G797-G806, 2010.

[85] P. M. Delaney, M. R. Harris, and R. G. King, "Novel microscopy using fibre optic confocal imaging and its suitability for subsurface blood vessel imaging in vivo," Clinical and Experimental Pharmacology and Physiology, vol. 20, no. 3, pp. 197-198, 1993.

[86] P. M. Delaney, M. R. Harris, and R. G. King, "Fiber-optic laser scanning confocal microscope suitable for fluorescence imaging," Applied Optics, vol. 33, no. 4, pp. 573-377, 1994.

[87] P. M. Delaney, R. G. King, J. R. Lambert, and M. R. Harris, "Fibre optic confocal imaging (FOCI) for subsurface microscopy of the colon in vivo," Journal of Anatomy, vol. 184, no. 1, pp. 157-160, 1994.

[88] M. Goetz, B. Memadathil, S. Biesterfeld et al., "In vivo subsurface morphological and functional cellular and subcellular imaging of the gastrointestinal tract with confocal minimicroscopy," World Journal of Gastroenterology, vol. 13, no. 15, pp. 2160-2165, 2007.

[89] W. J. McLaren, P. Anikijenko, S. G. Thomas, P. M. Delaney, and R. G. King, "In vivo detection of morphological and microvascular changes of the colon in association with colitis using fiberoptic confocal imaging (FOCI)," Digestive Diseases and Sciences, vol. 47, no. 11, pp. 2424-2433, 2002.

[90] M. Goetz, M. Vieth, S. Kanzler et al., "In vivo confocal laser laparoscopy allows real time subsurface microscopy in animal models of liver disease," Journal of Hepatology, vol. 48, no. 1, pp. 91-97, 2008.

[91] M. Goetz, J. V. Ansems, P. R. Galle, M. Schuchmann, and R. Kiesslich, "In vivo real-time imaging of the liver with confocal endomicroscopy permits visualization of the temporospatial patterns of hepatocyte apoptosis," American Journal of Physiology — Gastrointestinal and Liver Physiology, vol. 301, pp. G764-G772, 2011.

[92] M. Goetz, S. Thomas, A. Heimann et al., "Dynamic in vivo imaging of microvasculature and perfusion by miniaturized confocal laser microscopy," European Surgical Research, vol. 41, no. 3, pp. 290-297, 2008.

[93] A. J. M. Watson, S. Chu, L. Sieck et al., "Epithelial barrier function in vivo is sustained despite gaps in epithelial layers," Gastroenterology, vol. 129, no. 3, pp. 902-912, 2005.

[94] R. Kiesslich, M. Goetz, E. M. Angus et al., "Identification of epithelial gaps in human small and large intestine by confocal endomicroscopy," Gastroenterology, vol. 133, no. 6, pp. 1769$1778,2007$.

[95] J. J. Liu, K. L. Madsen, P. Boulanger, L. A. Dieleman, J. Meddings, and R. N. Fedorak, "Mind the gaps: confocal endomicroscopy showed increased density of small bowel epithelial gaps in inflammatory bowel disease," Journal of Clinical Gastroenterology, vol. 45, no. 3, pp. 240-245, 2011.

[96] J. J. Liu, K. Wong, A. L. Thiesen et al., "Increased epithelial gaps in the small intestines of patients with inflammatory bowel disease: density matters," Gastrointestinal Endoscopy, vol. 73, no. 6, pp. 1174-1180, 2011.

[97] D. Moussata, M. Goetz, A. Gloeckner et al., "Confocal laser endomicroscopy is a new imaging modality for recognition of intramucosal bacteria in inflammatory bowel disease in vivo," Gut, vol. 60, no. 1, pp. 26-33, 2011.

[98] R. Kiesslich, C. A. Duckworth, D. Moussata et al., "Local barrier dysfunction identified by confocal laser endomicroscopy predicts relapse in inflammatory bowel disease," Gut, vol. 61, pp. 1146-1153, 2012.

[99] A. Meining and M. B. Wallace, "Endoscopic imaging of angiogenesis in vivo," Gastroenterology, vol. 134, no. 4, pp. 915-918, 2008.

[100] M. Goetz and T. D. Wang, "Molecular imaging in gastrointestinal endoscopy," Gastroenterology, vol. 138, no. 3, pp. 828.e1-833.e1, 2010.

[101] U. Mahmood, "Optical molecular imaging approaches in colorectal cancer," Gastroenterology, vol. 138, no. 2, pp. 419422, 2010.

[102] P. L. Hsiung, J. Hardy, S. Friedland et al., "Detection of colonic dysplasia in vivo using a targeted heptapeptide and confocal microendoscopy," Nature Medicine, vol. 14, pp. 454458, 2008.

[103] M. Goetz, A. Ziebart, S. Foersch et al., "In vivo molecular imaging of colorectal cancer with confocal endomicroscopy by targeting epidermal growth factor receptor," Gastroenterology, vol. 138, no. 2, pp. 435-446, 2010.

[104] S. Foersch, R. Kiesslich, M. J. Waldner et al., "Molecular imaging of VEGF in gastrointestinal cancer in vivo using confocal laser endomicroscopy," Gut, vol. 59, pp. 1046-1055, 2010 . 
[105] C. Fottner, E. Mettler, M. Goetz et al., "In vivo molecular imaging of somatostatin receptors in pancreatic islet cells and neuroendocrine tumors by miniaturized confocal laserscanning fluorescence microscopy," Endocrinology, vol. 151, no. 5, pp. 2179-2188, 2010.

[106] M. S. Hoetker, R. Kiesslich, M. Diken et al., "Molecular in vivo imaging of gastric cancer in a human-murine xenograft model: targeting epidermal growth factor receptor (EGFR)," Gastrointestinal Endoscopy, vol. 76, pp. 612-620, 2012.

[107] M. S. Hoetker, R. Kiesslich, M. Diken, P. R. Galle, and M. Goetz, "Molecular endomicroscopy predicts tumor response to cetuximab therapy in human colon cancer xenografts," Gastroenterology, vol. 142, p. S-6, 2012. 


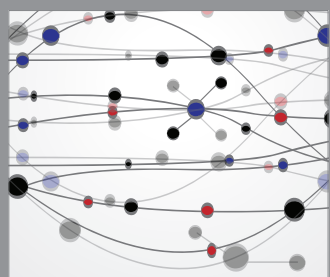

The Scientific World Journal
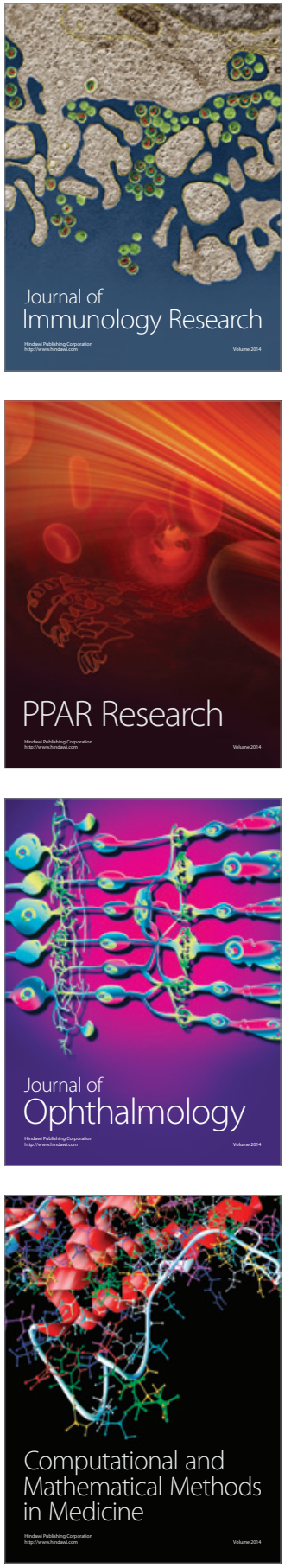

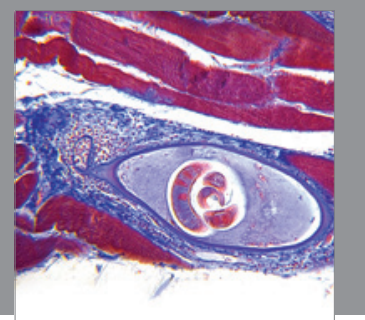

Gastroenterology

Research and Practice
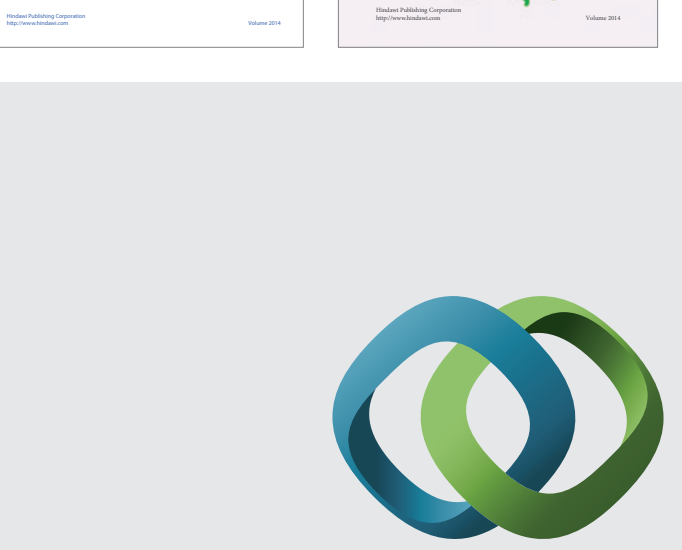

\section{Hindawi}

Submit your manuscripts at

http://www.hindawi.com
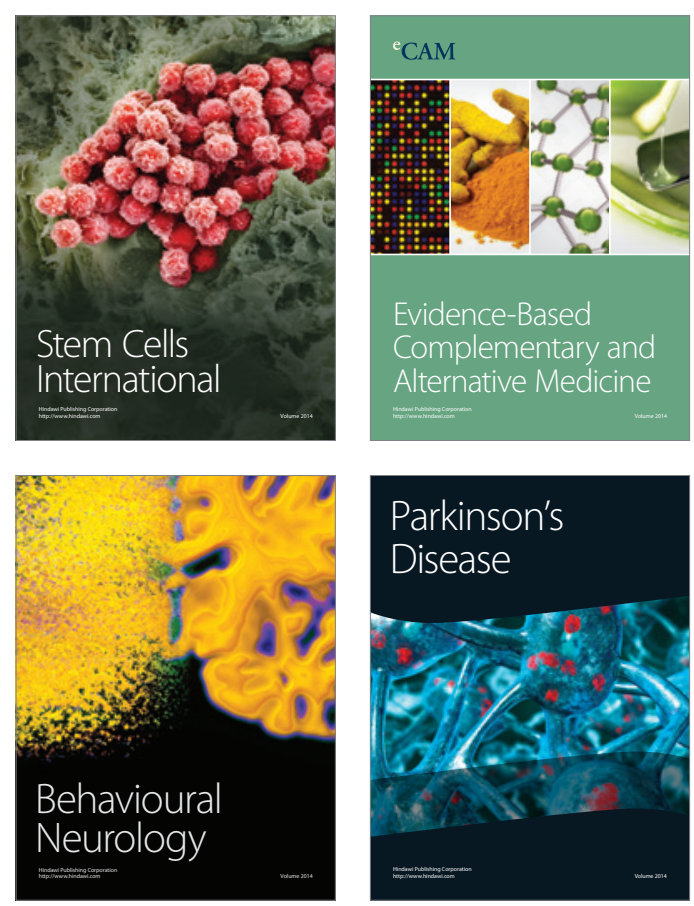

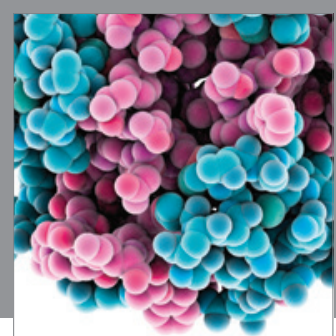

Journal of
Diabetes Research

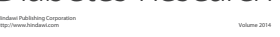

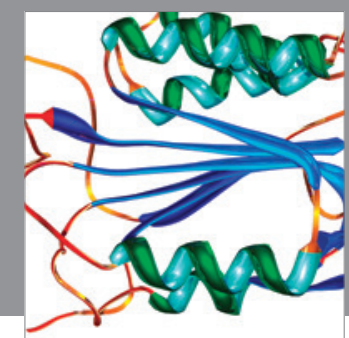

Disease Markers
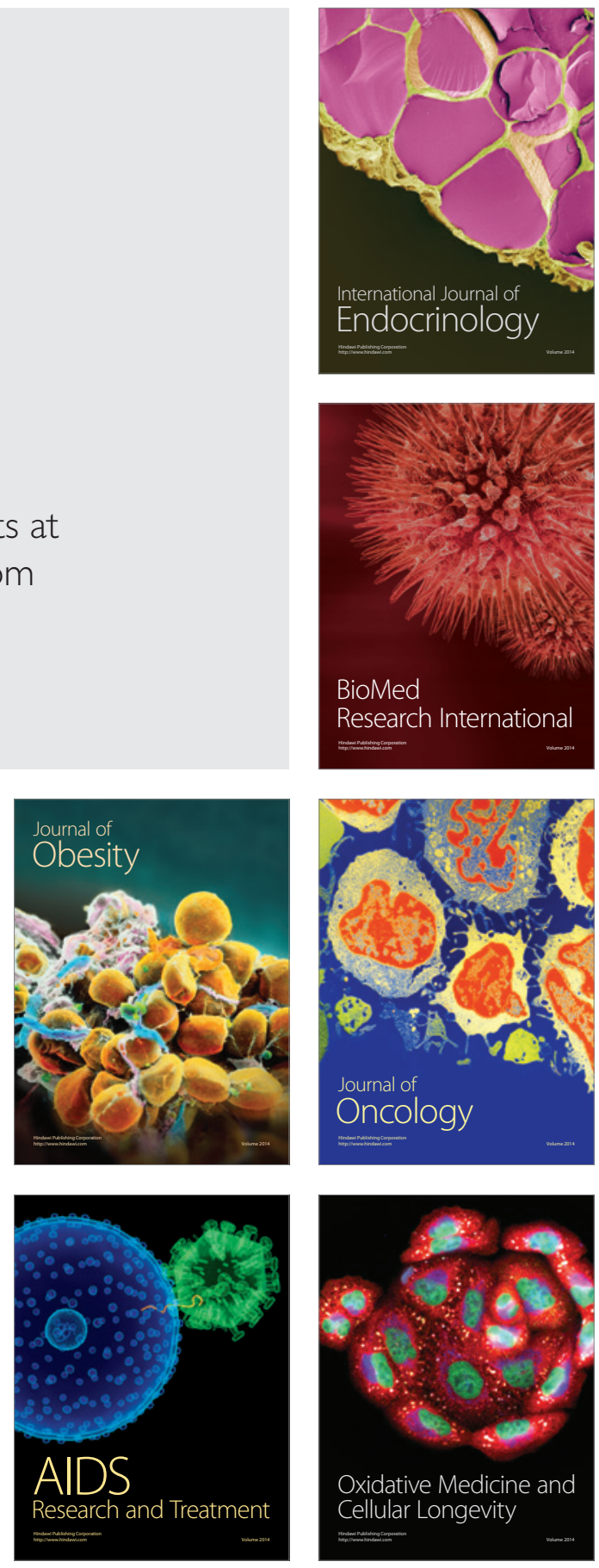